\title{
Evidence of two VHE gamma-ray sources in the W51 region
}

\section{Cyril Trichard*}

LAPP, Université Savoie Mont-Blanc, CNRS/IN2P3, Annecy-le-Vieux, France

E-mail: Cyril.trichardelapp.in2p3.fr

\section{Armand Fiasson,}

LAPP, Université Savoie Mont-Blanc, CNRS/IN2P3, Annecy-le-Vieux, France

E-mail: armand.fiassonelapp.in2p3.fr

\section{Vincent Marandon,}

Max-Planck-Institut für Kernphysik, Heidelberg, Germany

E-mail: vincent.marandon@mpi-hd.mpg.de

\section{on behalf of the H.E.S.S. Collaboration}

W51C is a supernova remant (SNR) known to be interacting with a molecular cloud (MC). Gamma rays with energies of hundreds of $\mathrm{MeV}$ up to tens of $\mathrm{TeV}$ were discovered towards this region. A spatially-coincident pulsar wind nebula (PWN) has previously hindered efforts to investigate cosmic ray acceleration at the SNR/MC shock. For the first time, thanks to improved data analysis methods, H.E.S.S. (High Energy Stereoscopic System) reveals a two-component gamma-ray source morphology in this region. Distinct emission from the SNR/MC interaction region and the PWN are observed, allowing very-high-energy (VHE; $E>0.1 \mathrm{TeV}$ ) gamma-ray spectra to be mesured from these two components. The latest H.E.S.S. results will be shown and compared to the latest observations by Fermi-LAT.

The 34th International Cosmic Ray Conference,

30 July- 6 August, 2015

The Hague, The Netherlands

\footnotetext{
* Speaker.
} 